\title{
Transformation europäischer Governance im Bereich der Verkehrspolitik
}

\author{
Dieter Plehwe*
}

\section{Europäisierung, Governance und quantitative (Mess-)Verfahren}

Der grundsätzliche wissenschaftliche Streit über den Charakter beziehungsweise die Finalität der europäischen Integration - die Frage der Staatswerdung - erscheint in Anbetracht der Vertiefungs- und Erweiterungsschritte der 1980er und 1990er Jahre und im Hinblick auf die vielfältige europapolitische Realität vielen Beobachtern als realitätsfern. Zudem erfasst die alte Auseinandersetzung zwischen intergouvernementalistischen und neo-funktionalistischen Positionen, die sich auf einen Primat nationaler oder supranationaler Institutionen im Mehrebenensystem konzentriert hat, wichtige Aspekte der jüngeren Veränderung der Staatlichkeit nicht, die beispielsweise im Zuge der neoliberalen Privatisierungspolitik an Bedeutung gewannen. In den Debatten zum Übergang vom steuernden Produzentenstaat zum koordinierenden Gewährleistungsstaat (,governance") ist gegenüber alten Europadebatten weniger die Regelungsebene des verflochtenen Regierungssystems von Interesse als die Frage, wie staatliche und nichtstaatliche Akteure ihre Aufgaben für eine gute Ordnung wahrnehmen (können). ${ }^{1}$ Der staatstheoretische Polity-Fokus tritt in der jüngeren Integrationsforschung häufig hinter einen pragmatischen Policy-(Netzwerk-)Fokus zurück.

Mit dem Perspektivwechsel in der EU-Forschung einher geht eine Hinwendung zu quantitativen Verfahren, die in den vorherigen Phasen der europäischen Integrationsforschung gegenüber qualitativen Untersuchungsdesigns überwiegend keine große Rolle spielten. Mit zunehmender Ausdifferenzierung der europäischen Politik und der mittlerweile erreichten Reife des Forschungsgebietes wächst allenthalben auch das Bedürfnis nach einer Theorieentwicklung, die über die Hypothesenbildung hinaus testenden Verfahren größeren Raum lässt. David Coen beispielsweise hält den Forschungsstand zu supranationalen Einflusslogiken in der europäischen Verbändeforschung mittlerweile für hinreichend gesichert, um neben vergleichenden Fallstudien auch quantitative Studien zu Fragen der europäischen Interessenvertretung einzufordern. ${ }^{2}$

Das wachsende Bedürfnis nach verallgemeinerbarer und gesicherter Erkenntnis in der Europaforschung ist verständlich. Allerdings muss zu Fragen der Europäisierung im Allgemeinen und zur Frage der Europäisierung des Rechts im Besonderen sehr sorgfältig gearbeitet werden, wenn quantitative Verfahren gewählt werden. Sowohl Mängel bei verfügbaren Datensätzen als auch die Komplexität europapolitischer Zusammenhänge führen in diesem Feld häufig zu gravierenden Missverständnissen und Fehleinschätzungen, wenn vorschnell und allzu pragmatisch gerechnet wird. Die weite Verbreitung des 80-Prozent-Mythos, wonach die nationale Demokratie gefährdet sei, weil der bei Weitem überwiegende Teil der Rechtsetzung mittlerweile in Brüssel erfolge, basiert beispielsweise auf Berechnungen des Verhältnisses zwischen europä-

* Dr. Dieter Plehwe, Abteilung, Internationalisierung und Organisation', Wissenschaftszentrum Berlin für Sozialforschung.

1 Ingeborg Tömmel (Hrsg.): Die Europäische Union. Governance und Policy-Making, Politische Vierteljahresschrift Sonderheft 40/2007.

2 David Coen: Empirical and theoretical studies in EU lobbying, in: Journal of European Public Policy 3/2007, S. 333-345. 
ischem und nationalem Recht, die die europäische Rechtsetzung in vieler Hinsicht extrem verzerren. ${ }^{3}$ Sorgfältige wissenschaftliche Untersuchungen in verschiedenen Mitgliedstaaten verdeutlichen demgegenüber, dass der supranationale Einfluss sowohl innerhalb als auch zwischen Politikfeldern und im Zeitverlauf schwankt, wobei festzuhalten ist, dass längst nicht alle quantitativen und qualitativen Fragen beispielsweise im Hinblick auf die Vergleichbarkeit von europäischen und nationalen Rechtsakten/Gesetzen oder Klassifikationssystemen geklärt sind. ${ }^{4}$

Vor dem Hintergrund des grundlegenden Klärungsbedarfes im Hinblick auf das Verhältnis zwischen nationalem und europäischem Recht - bereits in der dichotomischen Unterscheidung verbergen sich theoretisch problematische Vorentscheidungen ${ }^{5}$ - wendet sich der folgende Beitrag zur Untersuchung der Europäisierung ${ }^{6}$ anhand der europäischen Rechtsentwicklung der Frage zu, ob und inwiefern die quantitative Entwicklung der supranationalen europäischen Rechtsetzung für sich genommen Aussagen über den Verlauf und den sich möglicherweise wandelnden Charakter der Europäisierung erlaubt. ${ }^{7}$ Eine exemplarische Analyse der Europäisierung der Rechtsetzung im Politikfeld Verkehr (kurz Verkehrsrechtsetzung) unter Verwendung von quantitativen Methoden dient auch der Bildung von Hypothesen zur Heterogenität der Europäisierung des Rechts. Vorab soll erläutert werden, dass ein solches Vorgehen einerseits nötig ist, weil bereits vorliegende quantitative Untersuchungen zum europäischen Recht erhebliche Mängel aufweisen, die aus einer mangelnden Kenntnis der europäischen Rechtsetzung sowie einem blinden Vertrauen in verfügbare Datenbanken resultieren. Zum anderen erscheint das entwickelte Verfahren geeignet, bestimmte Defizite im Hinblick auf die Untersuchung der Europäisierung zu verringern, die aus der Fokussierung jüngerer Studien auf Governance-Fragen resultieren.

\section{Das Europarecht als Gegenstand quantitativer Analysen: blindes Vertrauen und blinde Flecken}

US-amerikanische Integrationsforscher entwickelten in der zweiten Hälfte der 1990er Jahre einen neuen integrationstheoretischen Ansatz, der symptomatisch für den bereits angesprochenen Perspektivwechsel in der Europaforschung ist. Der sogenannte ,Supranationalismus-Ansatz' knüpft zwar explizit an die neo-funktionalistische Theorietradition an, gibt sich aber im Hinblick auf den Verlauf und das Ziel der Integrationsprozesse agnostisch und will vordringlich mit empirischen Arbeiten zu einem besseren Verständnis unterschiedlicher Integrationsdynamiken beitragen. ${ }^{8}$ Trotz eines bereits im Namen - in explizitem Gegensatz

3 Dieter Plehwe: Zahlenpolitik. Europäisches Recht und nationalstaatliche Gesetzgebung, in: WZB-Mitteilungen 3/2007, S. 7-11.

4 Annette Elisabeth Töller: Mythen und Methoden. Zur Messung der Europäisierung der Gesetzgebung des Deutschen Bundestages jenseits des 80-Prozent-Mythos, in: Zeitschrift für Parlamentsfragen 1/2008, S. 3-17.

5 Armin Höland: Die Rechtssoziologie und der unbekannte Kontinent Europa, in: Zeitschrift für Rechtssoziologie 2/1993, S. 177-189.

6 Unter Europäisierung wird hier demnach nicht die Auswirkung der europäischen Politik auf der nationalen Ebene (z.B. der europäischen Rechtsetzung auf die nationale Gesetzgebung) verstanden, sondern die Entwicklung, insbesondere die Zunahme (Breite, Dichte) der europäischen Regelungen als Element der Herausbildung von Governance-Institutionen auf europäischer Ebene. Zu unterschiedlichen Europäisierungskonzeptionen vgl. Johan P. Olsen: The Many Faces of Europeanization, in: Journal of Common Market Studies 5/2002, S. 921-952.

7 Der Beitrag beruht auf der Studie von Dieter Plehwe (unter Mitarbeit von Katja Walther): Transformation Europäischer Governance: (Europa)Rechtliche Dimensionen. Eine vergleichende quantitative Analyse heterogener Europäisierungsprozesse im Politikfeld Verkehr, WZB Discussion Paper SP III 2007-201 des Wissenschaftszentrums Berlin für Sozialforschung, Berlin 2007. Ich danke Katja Walther für die Überarbeitung der Grafiken für diesen Beitrag und kritische Hinweise.

8 Andreas Nölke: Supranationalismus, in: Hans-Jürgen Bieling/Marika Lerch (Hrsg.): Theorien der europäischen Integration, Wiesbaden 2005, S. 145-168. 
zum Intergouvernementalismus - offenkundig die supranationale Ebene betonenden Ansatzes geht es in der konkreten Forschung nicht zuletzt um die präzisere Erfassung der Varianz der Reichweite der Europäisierung etwa im Vergleich von Politikfeldern, Branchen oder Institutionen. ${ }^{9}$ Die sehr starke Zunahme der europäischen (Binnenmarkt-)Rechtsetzung seit Verabschiedung der Einheitlichen Europäischen Akte (1987) weckte in diesem Zusammenhang verständlicherweise besonderes Interesse.

Vor dem Hintergrund der expansiven Entwicklung des europäischen Rechts und der Koevolution europäischer Interessengruppen gelangten US-amerikanische Soziologen in einer ambitionierten, (vermeintlich) alle Politikfelder quantitativ vergleichenden Studie zu dem Ergebnis, dass es sich seit Anfang der 1990er Jahre bei den meisten Politikfeldern in Europa um ,supranationale', also nicht (mehr) um zwischenstaatliche handele. ${ }^{10}$ Lediglich die Finanzpolitik, Steuerpolitik, Fischerei und Regionalpolitik verbleiben den Autoren zufolge als zwischenstaatliche Felder, weil nur in diesen Politikfeldern auf eine relativ große Zahl von Rechtsakten eine relativ kleine Zahl von Interessengruppen komme. Demnach würden nationale staatlich-politische Verhandlungs- und Entscheidungszentren in der Europäischen Union nur noch in wenigen Feldern schwerer gegenüber den supra- und transnationalen Kompetenz- und Entscheidungszusammenhängen wiegen. Ein integrationstheoretisch-pragmatischer Forschungszugang führt hier im Zusammenspiel mit vorwiegend quantitativen Messverfahren zu offenkundig sehr weitreichenden qualitativen Aussagen. Deren Haltbarkeit wird unterdessen schon durch gravierende Mängel bei der angewendeten Methode sehr stark infrage gestellt. Beispielsweise werden die unterschiedlichen institutionellen Entscheidungsverfahren bei der Differenzierung zwischen intergouvernemental und supranational vernachlässigt, die ganze Politikfelder oder Teile von Politikfeldern betreffen, und es wird eine Übereinstimmung der offiziellen Klassifikation des Europarechts mit den Politikfeldzuständigkeiten in der Kommission und im Ministerrat unterstellt, die so keineswegs gegeben ist. Völlig unzureichend ist schließlich die Wahl eines sehr kurzen Zeitraumes (1992-1994), in dem die Zahl der Rechtsakte (gemäß EUR-Lex-Klassifikation) mit der Zahl von Interessengruppen (gemäß freiwilligen Surveyangaben, die nicht kontrolliert wurden) korreliert werden, um zur qualitativen Aussage , supranational 'oder, zwischenstaatlich ' zu gelangen. Weil nicht einmal der diesbezügliche Schwellenwert expliziert und erläutert wird, bleibt das Ergebnis unbegründet. Unverkennbar ist in diesem Fall, dass vorhandene und spezifisch vorstrukturierte Daten trotz einer Diskussion der Quellen letztlich das quantitative Untersuchungsverfahren in problematischer Weise formen und ein fragwürdiges Ergebnis bestimmen. Viele der von den Autoren selbst angesprochenen und verworfenen Einwände gegen die Datengrundlage und die gewählten Verfahren sind demgegenüber mehr als berechtigt. ${ }^{11}$

Ein kritisches Verständnis der ,Rechtsdaten ${ }^{612}$ schließt ohne gründliche Vorarbeiten den Vergleich von allen (beziehungsweise vielen) Politikfeldern aus mehreren Gründen aus. Die von den Autoren verwendete empirische Datenbasis (EUR-Lex) ist zwar durchaus nützlich, kann aber unter anderem aus folgenden Gründen nicht ohne Weiteres verwendet werden:

9 Wayne Sandholtz/Alec Stone Sweet (Hrsg.): European Integration and Supranational Governance, Oxford 1998.

10 Neil Fligstein/Jason McNichol: The Institutional Terrain of the European Union, in: Sandholtz/Stone Sweet: Supranational Governance, 1998, S. 59-91.

11 Siehe zur ausführlichen Kritik der Methode Plehwe: Transformation Europäischer Governance, 2007, S. 14-16.

12 Grundsätzlich problematischer (unvollständiger) als die Rechtsdatenbank EUR-Lex ist die Datengrundlage, auf die sich die Autoren bei der Berechnung der Verbändedaten stützen, weil nachweislich viele und darüber hinaus besonders wichtige Interessengruppen im Rahmen der von den Autoren ohne eigene Prüfung ,bereinigten' Surveystudien nicht berücksichtigt worden sind. Siehe Plehwe: Transformation Europäischer Governance, 2007, S. 92-109. 
(1) Die Anzahl europäischer Rechtsakte in einem Politikfeld bietet aufgrund der sehr unterschiedlichen Art der Rechtsakte keine hinreichende Grundlage, auf deren Basis vernünftige Schlussfolgerungen gezogen werden können. Werden Verordnungen, Richtlinien und Entscheidungen einheitlich gezählt und gleich gewichtet, wird die Zahl der geltenden Rechtsakte inflationiert, unter anderem weil die meisten Entscheidungen lediglich für einen Mitgliedstaat gelten.

(2) Eine (zu) streng an das offizielle Klassifikationssystem des EUR-Lex-Registers angelehnte Zählung der Rechtsakte ist nicht hinreichend, weil Politikfelder und das Klassifikationssystem von EUR-Lex keineswegs so vollständig korrespondieren, wie die Autoren es unterstellen. Beispielsweise müssen im Politikfeld Verkehr auch Rechtsakte zu transeuropäischen (Verkehrs-)Infrastrukturvorhaben berücksichtigt werden, die seit Anfang der 1990er Jahre überwiegend im EUR-Lex-Register 13 (Industriepolitik, Unterkategorie Transeuropäische Netze) registriert werden. Zur Identifikation der relevanten Rechtsakte bietet die offizielle Klassifikation eine Orientierung, es müssen aber weitere Schritte unternommen werden, wie die Ergänzung der Datengrundlage auf der Basis der Eurovoc-Deskriptoren.

(3) Nicht reflektiert werden im Rahmen einfacher quantitativer Analysen qualitative Messfragen, beispielsweise, dass bisweilen mehrere europäische Rechtsakte erforderlich sind, um jeweils kleine Europäisierungsfortschritte zu erzielen, wohingegen mitunter mit einem einzigen Rechtsakt ein europapolitischer ,Durchbruch' erzielt werden kann. Es ist durchaus problematisch, von der Anzahl der Rechtsakte (und Interessengruppen) zu einem bestimmten Zeitpunkt auf den Grad der Europäisierung beziehungsweise den Vorrang von Ebenen zu schließen.

Trotz der notwendigen und grundsätzlichen Vorbehalte gegenüber quantitativen Messverfahren ${ }^{13}$ wäre es jedoch voreilig, das Anliegen einer auch auf quantitativer Messung beispielsweise der Rechtsentwicklung beruhenden Analyse von Europäisierungsprozessen vollends abzulehnen. Weniger umfassend angelegte, dafür aber solidere Einzelstudien oder Studien, die mehrere (Teil-)Politikfelder im längeren Zeitverlauf vergleichen, könnten ein differenziertes Bild der Europäisierung im Hinblick auf unterschiedliche Governance-Typen liefern, wobei die Häufigkeit der verschiedenen zur Anwendung kommenden Rechtsakte als Indikator genutzt werden kann. Solche quantitativen Verfahren sind beispielsweise geeignet, universelle Thesen, etwa zu einem fundamentalen Wandel der Staatlichkeit in Europa, anzuzweifeln. Eine im Rahmen der europäischen Governance-Diskussion bisweilen aufgestellte Hypothese der generellen Verdrängung des autoritativen durch einen kooperativen Staat konnte durch Politikfelder vergleichende Studien zurückgewiesen werden. Die Unterscheidung von bindenden Rechtsakten (,hard law') und nicht bindenden Rechtsakten (,soft law ${ }^{\text {) }}$ als Indikator für die Governance-Typen ,Hierarchie“ und ,Koordination“ ermöglicht es zu zeigen, dass in der europäischen Umweltpolitik hierarchische Steuerung überwiegt, während in der Sozialpolitik koordinierende Steuerung vorherrscht. ${ }^{14}$ In der Umweltpolitik lässt sich mithin auf europäischer Ebene keine Entwicklung beobachten, die es erlaubt, einen Übergang vom Governance-Typ ,Hierarchie‘ zur ,Koordination` zu konstatieren. Im Gegenteil:

13 Siehe zur problematischen Konversion qualitativer in quantitative Unterscheidungen, zum Risiko des, degreeism‘ und anderen konzeptionellen Europäisierungsfragen Claudio M. Radaelli: Whither Europeanization? Concept stretching and substantive change, in: European Integration online Papers 8/2000.

14 Holger Bähr/Oliver Treib/Gerda Falkner: Von Hierarchie zu Kooperation? Zur Entwicklung von GovernanceFormen in zwei regulativen Politikfeldern der EU, in: Tömmel: Europäische Union, 2007, S. 92-115. 
Es kann auf die europäische Politik zurückgeführt werden, dass auf nationaler Ebene ein hierarchischer Steuerungsstil (wieder) gestärkt wurde. ${ }^{15}$

Prinzipiell stehen Wissenschaftler/-innen bei der Verwendung quantitativer Verfahren zur Erforschung der Europäisierung ,[...] vor der grundsätzlichen Entscheidung zwischen analytischer Tiefe und empirischer Breite [... “". ${ }^{16}$ Während die Unterscheidung von bindenden und nicht bindenden Rechtsakten eine grobe Unterscheidung von Governance-Typen erlaubt, bleiben weitere Möglichkeiten quantitativer Verfahren zur Vertiefung der Analyse im Hinblick auf die Europäisierungsentwicklungen bislang ungenutzt. Zwar präzisieren die $\mathrm{Au}-$ toren der bereits genannten Governance-Studie ${ }^{17}$ ihre Argumentation im Hinblick auf bindende Rechtsakte durch die Unterscheidung zwischen neuen Regeln einerseits und späteren Anpassungen, Anwendungsregeln oder geographischen Ausdehnungen andererseits (Deflation der Rechtsakte) und verweisen darüber hinaus zu Recht auf die Notwendigkeit beziehungsweise die Möglichkeit der Analyse von Teilpolitikfeldern. Weitere Optionen einer differenzierten quantitativen Analyse des europäischen Rechts ergeben sich jedoch erst, wenn nicht nur zwischen bindenden und nicht bindenden Rechtstypen, also den allgemeinsten ,Governance-Typen“ (,Hierarchie" versus ,Koordination') unterschieden wird, sondern auch zwischen verschiedenen Typen des bindenden Rechts und wenn darüber hinaus Teilpolitikfelder systematisch untersucht werden. Die Unterscheidung zwischen den verschiedenen Typen bindenden Rechts - insbesondere zwischen Verordnungen und Richtlinien - erscheint beispielsweise geeignet, die im Zuge der Governance-Diskussion in den Hintergrund geratene Europäisierungsfrage stärker in den Vordergrund zu rücken. Die Bedeutung der verschiedenen Formen hierarchischer Steuerung - unmittelbar bindendes supranationales Recht und prinzipiell nationalstaatlich vermitteltes Rahmenrecht - wird im Folgenden anhand einer Analyse des Politikfeldes Verkehr sowie relevanter Teilpolitikfelder im Bereich Verkehr (Branchen und Regelungsbereiche) veranschaulicht.

\section{Heterogene Europäisierung: eine exemplarische Analyse der Verkehrsrechtsetzung}

Das Forschungsinteresse der zuvor diskutierten quantitativen Studien ${ }^{18}$ richtete sich auf die Frage der Europäisierung unter Vernachlässigung der verschiedenen Rechts- und Governance-Typen oder auf die Frage vorherrschender Governance-Typen unter Vernachlässigung der Frage der Europäisierung. Anhand der Ergebnisse einer systematisch zwischen Rechtstypen und Teilpolitikfeldern unterscheidenden quantitativen Analyse des europäischen Verkehrsrechts kann demgegenüber gezeigt werden, dass die beiden Forschungsperspektiven besser integriert werden können, um den innerhalb und zwischen Politikfeldern in mehrfacher Hinsicht heterogenen Europäisierungsprozessen gerecht zu werden.

Gegenüber der These von der Vereinheitlichung der Verkehrspolitik zu einem supranationalen Politikfeld zeigt eine hinreichend differenzierte Analyse, dass die Verkehrspolitik zwar sukzessive in ein umfassend von supranationalen Regeln betroffenes Politikfeld verwandelt wurde. Jedoch können ungleichzeitige und in anderer Hinsicht ungleiche Europäisierungsdynamiken im Hinblick auf Branchen und Regelungsmaterien erkannt und

15 Annette Elisabeth Töller: Die Rückkehr des befehlenden Staates? Muster und Ursachen der Veränderung staatlicher Handlungsformen in der deutschen Abfallpolitik, in: Politische Vierteljahresschrift 1/2007, S. 66-96; Annette Elisabeth Töller: The Rise or Fall of the Co-operative State? On the Contingent Effects of European Law on German Environmental Policy, Center for European Studies (Harvard University), Program for the Study of Germany and Europe Working Paper Series 07.4/2007.

16 Bähr/Treib/Falkner: Von Hierarchie zu Kooperation?, 2007, S. 97.

17 Bähr/Treib/Falkner: Von Hierarchie zu Kooperation?, 2007.

18 Ebenda; Fligstein/McNichol: The Institutional Terrain, 1998. 
beschrieben werden. Im Gegensatz zur allgemeinen Unterscheidung von ,Koordination “ und ,Hierarchie“ als zentralen Governance-Typen erlaubt es eine weitere Differenzierung der hierarchischen Steuerung anhand von Richtlinien und Verordnungen (prinzipiell nationalstaatlich vermittelte gegenüber unmittelbar supranationaler ,Hierarchie'), wichtige Unterschiede im Hinblick auf Europäisierungsmodi zu erfassen. Um die Entwicklung der Europäisierung der Rechtsetzung in (Teil-)Politikfeldern angemessen zu beurteilen, muss allerdings über die offiziellen Klassifikationsgrenzen des Europarechts hinaus recherchiert werden, wobei beispielsweise auf die den Rechtsakten offiziell zugeordneten Eurovoc-Deskriptoren zurückgegriffen werden kann.

\section{Prozessperspektive: von ,Koordination' $z u$,Hierarchie‘}

Während in der Rechtsetzung zur europäischen Sozialpolitik unverbindliche Rechtsakte vorherrschen, die einen koordinierenden Politikstil anzeigen, und in der europäischen Umweltpolitik verbindliche Regeln überwiegen, weshalb von einem vorwiegend hierarchischen Politikstil gesprochen werden kann, zeigt die Entwicklung der europäischen Verkehrsrechtsetzung, dass sich hier bis in die frühen 1980er Jahre verbindliche und nicht verbindliche Regeln insgesamt in etwa die Waage hielten, seither aber verbindliche Regeln klar dominieren. Die folgende Grafik 1 veranschaulicht die Verteilung des bindenden und nicht bindenden Verkehrsrechts gemäß der offiziellen EUR-Lex-Klassifikation 7 ,Verkehr` im Zeitverlauf.

\section{Grafik 1: Europäische Verkehrsrechtsetzung 1960-2004}

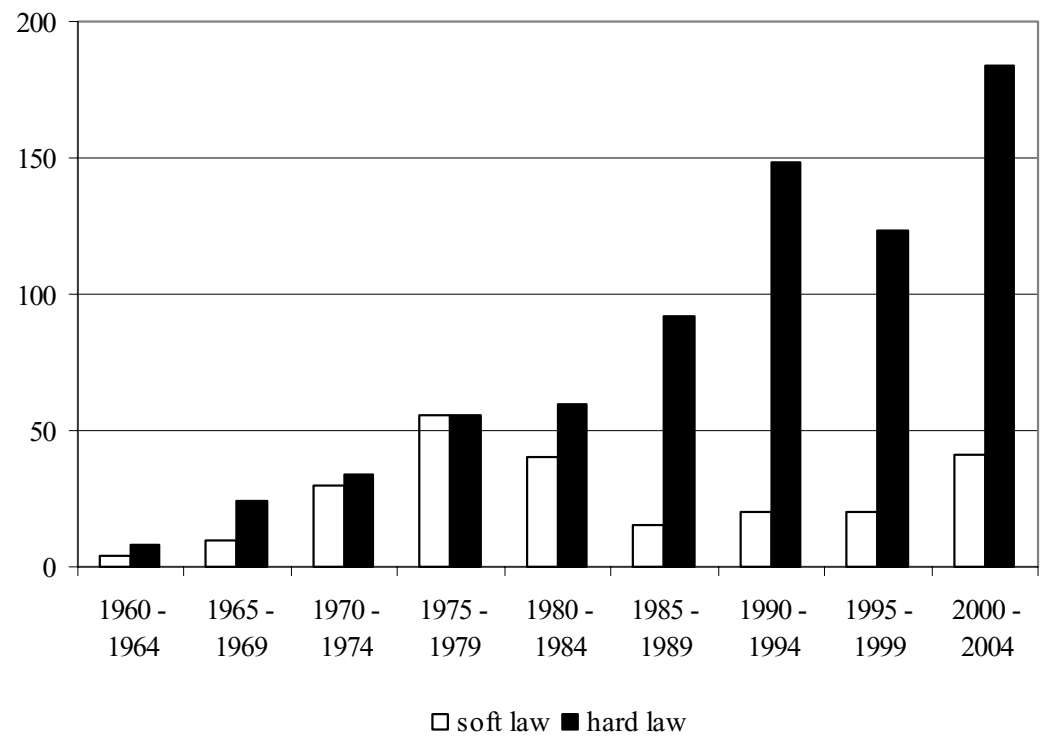

Quelle: EUR-Lex 7 (Stand Dezember 2005), Fünfjahresintervalle, eigene Berechnung.

Zwar überwogen auch in der frühen Phase der europäischen Verkehrsrechtsetzung meist die verbindlichen Regeln, aber aufgrund der bis in die 1980er Jahre hinein geltenden Harmonisierungspriorität in der gemeinsamen Verkehrspolitik wurden sehr häufig unverbindliche Instrumente, zum Beispiel Mitteilungen der Kommission zu nationalen Gesetzen, ${ }^{19}$ 
genutzt, obwohl für den Inlandsverkehr (Straße, Schiene, Binnenwasserwege) bereits in den Römischen Verträgen (als einzigem Gebiet neben der Agrarpolitik) die höchste Stufe der Integration vereinbart worden war. Erst nachdem die bis Mitte der 1980er Jahre in den Mitgliedstaaten vorherrschende Interpretation der gemeinsamen Verkehrspolitik, die eine Harmonisierung in vielen technischen und sozialen Bereichen als Vorbedingung von Liberalisierungsschritten ansah, vom Europäischen Gerichtshof revidiert wurde, rückten bis dahin vernachlässigte Vertragsziele der gemeinsamen Verkehrspolitik, insbesondere die Schaffung eines gemeinsamen europäischen Inlandsverkehrsmarktes, auf die europapolitische Agenda. Eingeführt wurde nun für den Inlandsverkehr auch das eigentlich sehr viel früher vorgesehene Abstimmungsverfahren mit qualifizierter Mehrheit. Bei der Umsetzung der neuen Agenda wurde in der Folgezeit, wie Grafik 1 verdeutlicht, in erheblich stärkerem Maße bindendes europäisches Recht verabschiedet.

Darüber hinaus entwickelte die Europäische Kommission neue Strategien, die vertragsrechtlich nicht in die gemeinsame Verkehrspolitik eingegliederten Bereiche des Hochseeund Luftverkehrs (Souveränitätsvorbehalt) verstärkt und in erheblichem Maße zu europäisieren. Obwohl sich die Mitgliedstaaten bei der Regulierung der entsprechenden Branchen bis heute Vetorechte vorbehalten, war die Kommission diesbezüglich insbesondere aufgrund ihrer wettbewerbsrechtlichen Kompetenzen und mit erheblicher Unterstützung durch die Rechtsprechung des Europäischen Gerichtshofes durchaus erfolgreich. ${ }^{20}$ Die folgende Grafik 2 dokumentiert, dass bis Mitte der 1980er Jahre der See- und Luftverkehr sowie die Transportwege (Infrastruktur) nur selten Gegenstand verbindlicher Rechtsetzung auf europäischer Ebene waren, seither aber häufig durch EG-Rechtsetzung geregelt werden.

Bedenkt man, dass sich hinter der Kategorie ,Inlandstransport' verschiedene Verkehrsträger und Branchen verbergen (Straßenverkehr, Schienenverkehr, Binnenschifffahrt), so deuten die Informationen in Grafik 2 an, dass mittlerweile kein eklatanter Unterschied mehr herrscht zwischen den großen Verkehrsbereichen im Hinblick auf die Häufigkeit der Betroffenheit von verbindlicher europäischer Rechtsetzung. Diese Aussage gilt im Übrigen auch für die Infrastrukturrechtsetzung, weil die auf die offizielle Klassifikation EUR-Lex 7 beschränkte Darstellung in Grafik 2 in die Irre führt: Seit Anfang der 1990er Jahre wurde die Rechtsetzung zu Transeuropäischen Netzen, darunter die Verkehrsnetze, in der offiziellen EUR-Lex-Klassifikation 13 ,Industriepolitik' rubriziert. Werden die dort gespeicherten Rechtsakte zu transeuropäischen Verkehrsnetzen ergänzt, so erhöht sich die Zahl der zwischen 1990 und 2005 zu berücksichtigenden Rechtsakte immerhin um 30. ${ }^{21}$ Der ungewöhnliche Spitzenwert für 1985 (16 europäische Infrastrukturrechtsakte) veranschaulicht demgegenüber eine bereits angesprochene Problematik der quantitativen Analyse des europäischen Rechts. Es handelte sich im Jahr 1985 um verbindliche Entscheidungen des Ministerrats zu jeweils nationalen Vorhaben, also nicht um für alle Mitgliedstaaten geltende Regelungen, wie sie etwa durch Richtlinien oder Verordnungen getroffen werden. ${ }^{22}$ Bei der seit den

19 Weil beispielsweise Mitteilungen der Kommission und Entscheidungen des Rates sich häufig an eine bestimmte Regierung richten, wird die europäische Rechtsetzung gewissermaßen ,inflationiert".

20 Zur Entwicklung der europäischen Verkehrspolitik, insbesondere auch zur Untätigkeitsklage gegen den Ministerrat und zum diesbezüglichen Urteil des Europäischen Gerichtshofes siehe Dieter Plehwe: Eurologistik, »Europäische« Verkehrspolitik und die Entwicklung eines transnationalen (Güter-)Transportsystems, in: Prokla 2/1997, S. 217-243.

21 Plehwe: Transformation Europäischer Governance, 2007, S. 57-58.

22 Auf die Problematik der Zählung von Entscheidungen machen auch Bähr/Treib/Falkner: Von Hierarchie zu Kooperation?, 2007, S. 96 aufmerksam. Allerdings können Entscheidungen nicht vollständig ausgeschlossen werden, weil sie zwar in der Regel nicht für alle Mitgliedstaaten gelten, aber durchaus nicht selten Ausnahmen von dieser Regel gelten (zum Beispiel in der europäischen Verkehrstechnologiepolitik). 
1990er Jahren zu beobachtenden Zunahme der europäischen Infrastrukturrechtsetzung handelt es sich dagegen überwiegend um Richtlinien. Entscheidend für das Wachstum der für alle Mitgliedstaaten verbindlichen Rechtsetzung in diesem Teilpolitikfeld ist, dass der Europäischen Kommission mit dem Maastrichter Vertrag eine neue Kompetenz im Bereich der Transeuropäischen Netze übertragen wurde.

\section{Grafik 2: Europäische Verkehrsrechtsetzung 1960-2004, hard law, nach Bereichen}

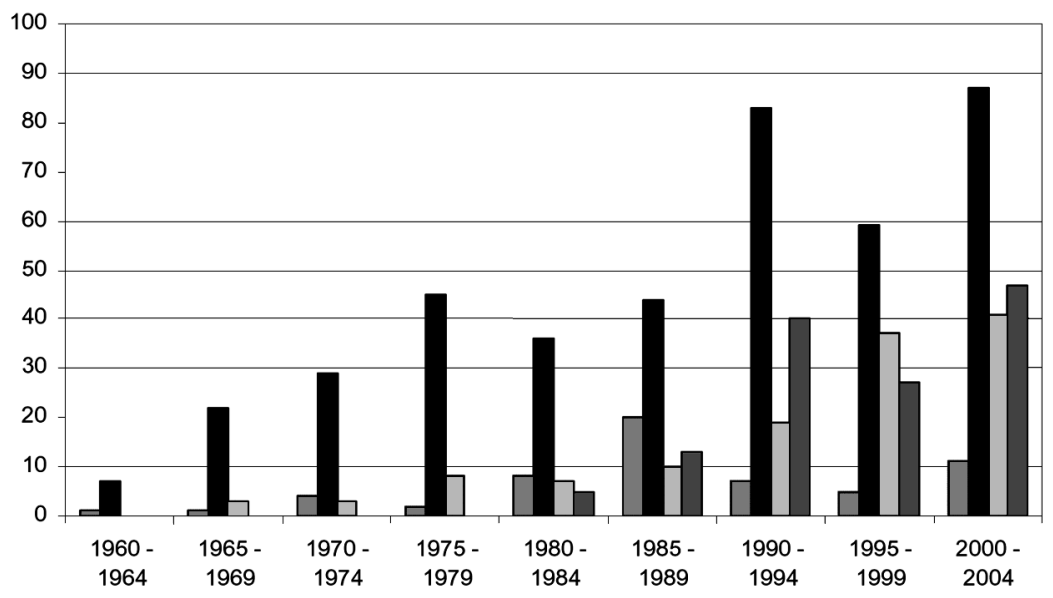

$\square$ Transportinfrastruktur $\square$ Inlandstransport $\square$ Seeverkehr $\square$ Luftverkehr

Quelle: EUR-Lex 7 (Stand Dezember 2005), eigene Berechnung.

Aus den beiden zunächst differenziert berechneten Entwicklungen lässt sich schließen, dass sich der vorherrschende Governance-Modus der gemeinsamen Verkehrspolitik in den 1980er Jahren geändert hat, wobei die hierarchischen Steuerungsformen deutlich verstärkt wurden und sukzessive auf alle großen Bereiche (Verkehrsträger und -branchen) ausgedehnt worden sind. Was spricht vor diesem Hintergrund dagegen, sich der Einschätzung der diskutierten Studie anzuschließen, der zufolge es sich bei der Verkehrspolitik um ein supranationales Politikfeld handelt? Zum einen vernachlässigt diese Einschätzung die Tatsache, dass in der Verkehrspolitik im Hinblick auf verschiedene Verkehrsträger und -branchen bis heute unterschiedliche Entscheidungs- und Verhandlungsverfahren gelten. Der im Hinblick auf den Luftverkehr und die Hochseeschifffahrt weiter bestehende institutionelle Einigungszwang (zumindest formal) unter Berücksichtigung der Position jeder einzelnen Regierung eines Mitgliedstaates - das Vetorecht als harter Kern zwischenstaatlicher (internationaler) Politik - wird offenkundig sehr gering geschätzt, wenn die Verkehrsrechtsetzung gleichwohl einheitlich eingeschätzt wird. Insbesondere die unterschiedlichen Mechanismen der Europäisierung, beispielsweise das Eigengewicht supranationaler Institutionen im Hinblick auf das Wachstum der Luftverkehrsrechtsetzung trotz Einstimmigkeitsverfahren im Gegensatz zum von den Mitgliedstaaten geförderten Wachstum der Inlandsverkehrsrechtsetzung unter der Bedingung von Mehrheitsentscheidungen, fallen unter den Tisch. Zum anderen verdeckt auch der allgemeine Eindruck des beachtlichen Wachstums verbindlicher europäischer (Verkehrs-)Rechtsetzung in allen Bereichen erhebliche Unterschiede zwischen verschiede- 
nen Branchen und Regelungsmaterien. Diese können sichtbar gemacht werden, indem genauer zwischen den verschiedenen Typen des bindenden Rechts unterschieden wird. Dabei ist vorab grundsätzlich anzumerken, dass die Europäisierung der Güterverkehrsrechtsetzung in mancher Hinsicht erheblich weiter fortgeschritten ist als die Europäisierung der Personenverkehrsrechtsetzung. Also kann die Europäisierung des Politikfeldes Verkehr auch diesbezüglich keineswegs einheitlich eingeschätzt werden. ${ }^{23}$ Im Folgenden wird die Analyse deshalb auf die quantitative Entwicklung der Rechtsetzung im Güterverkehr eingeschränkt.

\section{Branchen und Regelungsbereiche: verschiedene Europäisierungstypen}

Eine quantitative Analyse der Marktordnungsrechtsetzung im Güterverkehr zeigt insgesamt zunächst ein der allgemeinen Entwicklung der Verkehrsrechtsetzung entsprechendes Bild starken Wachstums. Allerdings unterbricht die besonders hohe Zahl der bindenden Güterverkehrsmarktrechtsakte in der ersten Hälfte der 1990er Jahre das Bild der Kontinuität und in der jüngsten Zeit wuchs die Zahl der nicht bindenden Rechtsakte besonders stark (siehe Grafik 3).

\section{Grafik 3: Europäische Güterverkehrsmarktrechtsetzung 1960-2004}

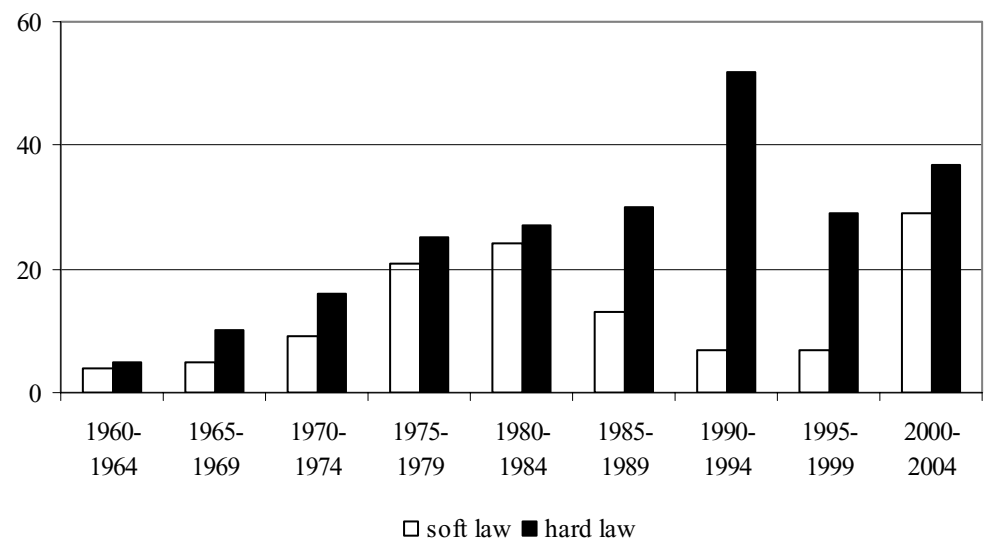

Quelle: EUR-Lex 7 (Stand Dezember 2005), Fünfjahresintervalle, eigene Berechnung.

Dies könnte bedeuten, dass nach einer Phase maßgeblich hierarchischer Steuerung wieder stärker auf Koordination gesetzt wird. Allerdings handelte es sich im vorliegenden Fall zwischen 2000 und 2004 überwiegend um nicht bindende Rechtsakte im Luftverkehr, die schließlich in bindende Regelungen mündeten, also eher um eine partikulare Anomalie. Generell sollte das Absinken der Zahl der Rechtsakte nicht vorschnell als Ausdruck einer schwächeren Europäisierung oder gar einer ,Re-Nationalisierung ' begriffen werden, weil beispielsweise das zuvor erreichte Niveau der Europäisierung nicht weiterentwickelt werden kann und jedenfalls nicht zurückgeschraubt wird. Im Hinblick auf einen gemeinsamen euro-

23 Die Vorschläge zur Liberalisierung des (grenzüberschreitenden und nationalen) öffentlichen Personenverkehrs sind jüngsten Datums und sehen zum Beispiel im Fall der Liberalisierung des nationalen Eisenbahnpersonenverkehrs einen Zeithorizont bis 2017 vor. Zur schwierigen Unterscheidung der Personen- und Güterverkehrsrechtsetzung unter Verwendung der Eurovoc-Deskriptoren (Verschlagwortung) siehe den diesbezüglichen Exkurs in Plehwe: Transformation Europäischer Governance, 2007, S. 26-27. 
päischen Verkehrsmarkt sind die Liberalisierungsziele weitgehend erreicht, wird der bereits erreichte Grad der grenzüberschreitenden Liberalisierung jedenfalls bislang nicht revidiert und auch in der Zukunft ist diese Option sehr unwahrscheinlich.

Markante Unterschiede in der Marktordnungsrechtsetzung für die verschiedenen Branchen treten unterdessen hervor, wenn systematisch zwischen den verschiedenen Typen des bindenden Rechts unterschieden wird. Die folgende Grafik 4 zeigt zunächst die sich ergebenden Werte für die gesamte Marktordnungsrechtsetzung.

\section{Grafik 4: Europäische Güterverkehrsmarktrechtsetzung 1960-2004, hard law}

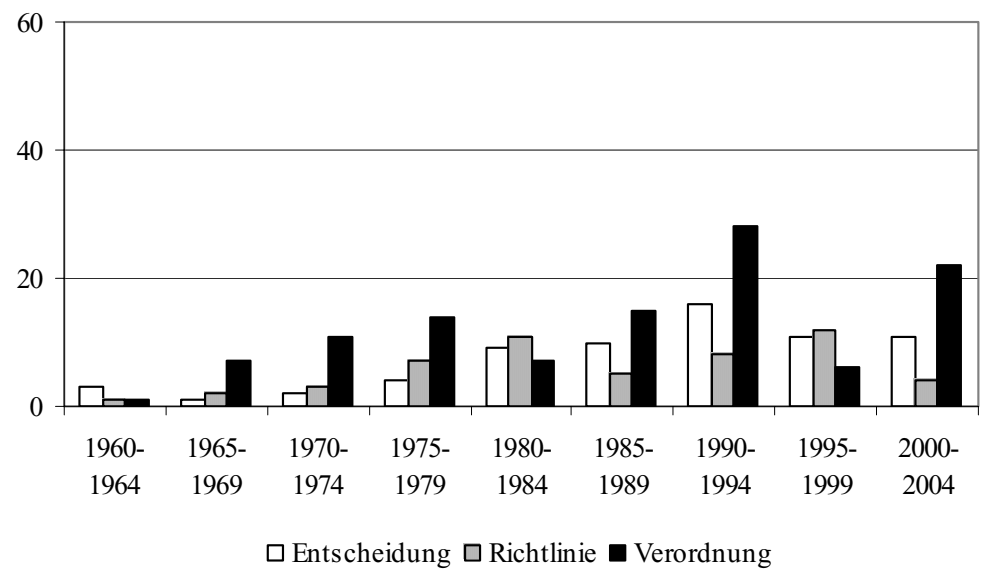

Quelle: EUR-Lex 7 (Stand Dezember 2005), Fünfjahresintervalle, eigene Berechnung.

Werden die Entscheidungen vernachlässigt, weil sie sich in der Regel auf einzelne Mitgliedstaaten beziehen, so sticht hervor, dass mit Ausnahme der Perioden 1980-1984 und 1995-1999 die Zahl der Verordnungen gegenüber der Zahl der Richtlinien überwiegt. Unter der Annahme, dass Verordnungen den stärksten Europäisierungsmodus anzeigen, weil sie in den Mitgliedstaaten unmittelbar Geltung erlangen, während Richtlinien als Rahmenrechtsetzung erst dann wirksam werden, wenn sie auf nationaler Ebene umgesetzt worden sind (und dabei mehr oder weniger große Spielräume durch den nationalen Gesetzgeber genutzt werden können ${ }^{24}$ ), ist es interessant, die allgemeinen Angaben mit den Zahlen für die verschiedenen Branchen zu kontrastieren. Exemplarisch zeigen wir im Folgenden die Werte für die Inlandsverkehrsbereiche Straßengüterverkehr (Grafik 5) und Eisenbahn (Grafik 6) sowie für den Luftverkehr (Grafik 7).

24 Mit den , direct effect ' und ,indirect effect ' Doktrinen des EuGH wurde die Wirksamkeit der europäischen Richtlinienrechtsetzung prinzipiell erhöht, vgl. Alec Stone Sweet/James A. Caporaso: From Free Trade to Supranational Polity: The European Court and Integration, in: Sandholtz/Stone Sweet: Supranational Governance, 1998, S. 92-133. 
Grafik 5: Straßengüterverkehr - europäische Güterverkehrsmarktrechtsetzung 19602004, hard law

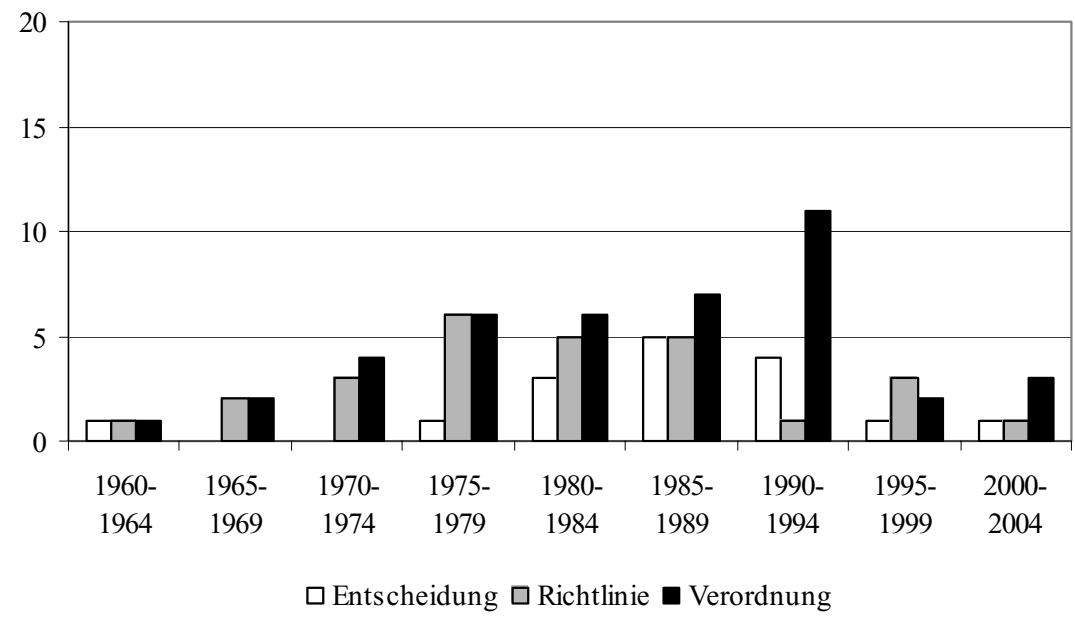

Quelle: EUR-Lex 7 (Stand Dezember 2005), Fünfjahresintervalle, eigene Berechnung.

Auf den Straßengüterverkehr entfällt der größte Teil der Verkehrsmarktrechtsetzung. Seit den frühen 1980er Jahren überwiegen die Verordnungen mit Ausnahme des Zeitraums von 1995-1999. Demgegenüber zeigt die folgende Grafik 6, dass Marktordnungsrechtsakte im Bereich der Eisenbahnen insgesamt selten sind und bislang die Zahl der Richtlinien die Zahl der Verordnungen deutlich übersteigt.

Grafik 6: Eisenbahnverkehr - europäische Güterverkehrsmarktrechtsetzung 19602004, hard law

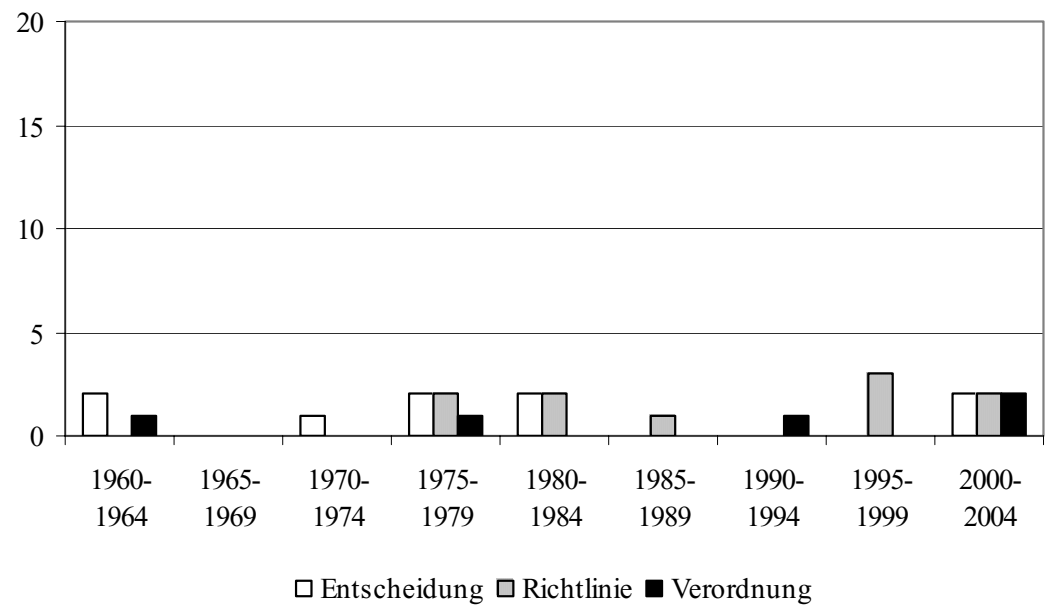

Quelle: EUR-Lex 7 (Stand Dezember 2005), Fünfjahresintervalle, eigene Berechnung. 
Im Luftverkehr nahm die Marktordnungsrechtsetzung seit Anfang der 1990er Jahre stark zu, wobei insgesamt viel stärker auf Verordnungen zurückgegriffen wurde als auf Richtlinien.

\section{Grafik 7: Luftverkehr - europäische Güterverkehrsmarktrechtsetzung 1960-2004, hard law}

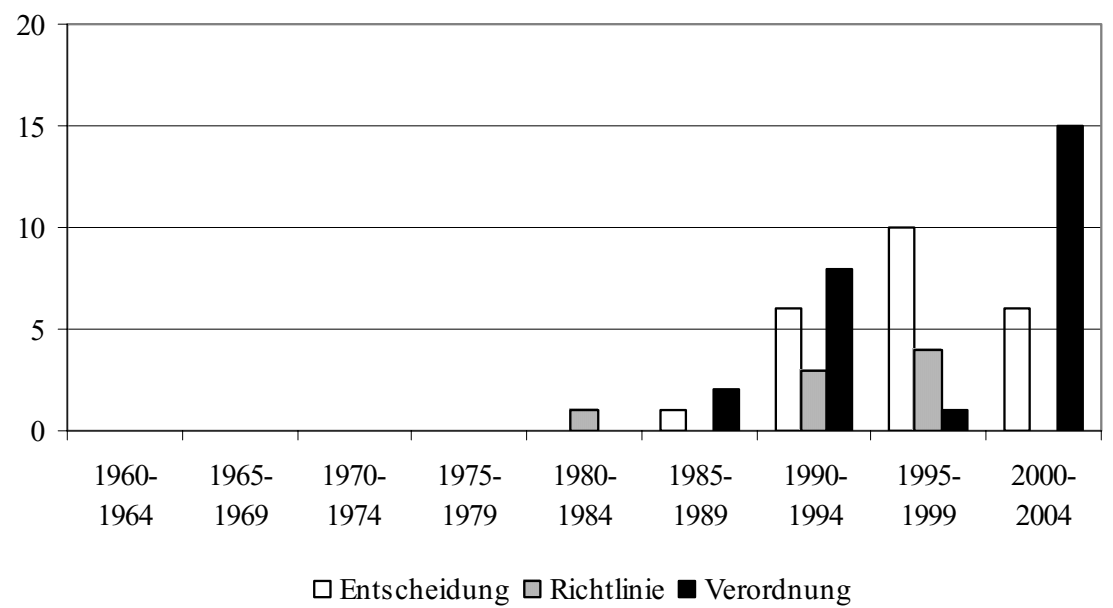

Quelle: EUR-Lex 7 (Stand Dezember 2005), Fünfjahresintervalle, eigene Berechnung.

Verkehrsexperten dürfte das Ergebnis im Hinblick auf den Eisenbahnsektor kaum überraschen. Tatsächlich sind in diesem Bereich außergewöhnliche und vielfältige (technischorganisatorische, politisch-rechtliche) Hürden für die recht schwerfällige Europäisierung verantwortlich. Es dürfte im Bereich der Eisenbahnpolitik kaum Probleme bereiten, einen durch die Richtlinienvorherrschaft angezeigten und jedenfalls gegenüber dem Straßengüterverkehr sehr viel stärker rekursiven Europäisierungstyp nationalstaatlicher Prägung zu verifizieren. ${ }^{25}$ Gleichzeitig mahnt die Entwicklung der europäischen Marktordnungsrechtsetzung im Eisenbahnverkehr aber auch zur Vorsicht im Hinblick auf eine zu starke Bewertung der Unterscheidung von Richtlinien und Verordnungen: Die nationalstaatlichen Spielräume bei der Gestaltung der Eisenbahnmarktordnung konnten nämlich sukzessive mithilfe von europäischen Richtlinien eingeschränkt werden. Erst eine qualitativ vertiefte Forschung erlaubt die inhaltliche Deutung der verwendeten rechtlichen Maßnahmen. Aber ein systematischer Vergleich des in allen Mitgliedstaaten gleichermaßen geltenden europäischen Rechts (Verordnungen) mit dem europäischen Rahmenrecht (Richtlinien) bietet sich immerhin zur Bildung von Hypothesen über den vorherrschenden Europäisierungstyp an, weil entweder nationale Prägungen der Politik in höherem Maße ausgeschlossen oder explizit zugelassen werden, also umfassende Konvergenz oder beschränkte (kompatible) Heterogenität angestrebt wird. Diesbezügliche Entwicklungen im Hinblick auf andere Regelungsmaterien zeigen, dass beispielsweise in den Bereichen der Verkehrssozial- und der Verkehrsumweltpoli-

$25 \mathrm{Ob}$ die strategischen Bemühungen unter anderem der Europäischen Kommission dabei normativ vor allem dahingehend zu interpretieren sind, dass nationale Blockaden aufgebrochen werden, sei dahin gestellt. Siehe dazu: Christoph Knill/Dirk Lehmkuhl: An Alternative Route of Legal Integration: The Community ss Railways Policy, in: European Integration Online Papers 3/1998. 
tik gegenüber der Marktordnungspolitik im Verkehr insgesamt vorwiegend Richtlinien zur Anwendung kommen. Abschließend sollen diese beiden Teil-Politikfelder etwas genauer erörtert werden, unter anderem auch weil die jüngste Entwicklung eine Veränderung des Europäisierungsmodus in diesen Bereichen andeuten könnte.

Im Gegensatz zur oben angesprochenen These eines vorherrschenden Governance-Typs ,Koordination' im Bereich der Sozialpolitik zeigt eine detaillierte Analyse der Sozialrechtsetzung für den Bereich Verkehr, ${ }^{26}$ dass in diesem Teilgebiet der Sozialpolitik seit Mitte der 1980er Jahre das bindende Recht bei Weitem überwiegt.

Wie Grafik 8 zeigt, war der Verkehr bereits früh ein Politikfeld, in dem die Sozialpolitik eine große Rolle gespielt hat. Allerdings kann anhand der Informationen in dieser Grafik ein bereits angesprochenes Problem quantitativer Verfahren näher erläutert werden, wenn mit einer großen beziehungsweise steigenden Zahl von Rechtsakten stärkere Europäisierungsprozesse assoziiert werden. Während für die Gesamtheit aller Arbeitnehmer/-innen Anfang der 1990er Jahre ein einziger europäischer Rechtsakt zur Arbeitszeitregulierung genügte, um europäische Mindeststandards (Urlaub, Ruhezeiten, maximale Arbeitszeiten etc.) zu setzen, wurden für die zunächst von der europäischen Arbeitszeitrichtlinie ausgenommenen Branchen des Verkehrs jeweils eigene und gegenüber der generellen Richtlinie zum Teil stark verwässerte Richtlinien verabschiedet. Im Fall der Arbeitszeitrichtlinien im Verkehr kommt mit der Anzahl der Rechtsakte mithin eher eine spezifische Europäisierungsproblematik im Verkehr zum Ausdruck (Verschleppung, Senkung der Standards) als eine ,starke 'Europäisierung per se.

\section{Grafik 8: Europäische Verkehrssozialrechtsetzung 1960-2004}

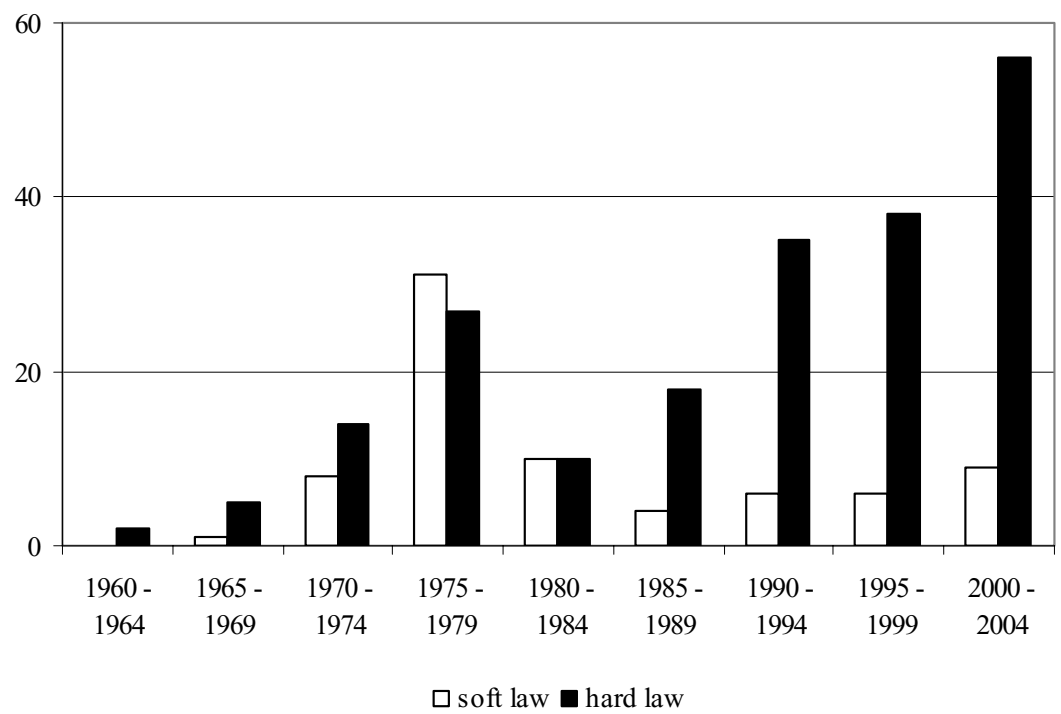

Quelle: EUR-Lex 7 und 5 (Stand Dezember 2005), Fünfjahresintervalle, eigene Berechnung.

26 Abgesehen von 183 Rechtsakten in EUR-Lex 7 ,Verkehr` konnten 136 Rechtsakte in EUR-Lex 5 , Arbeitnehmerfreizügigkeit und Sozialpolitik‘ identifiziert werden, die für die Verkehrspolitik relevant sind. Zum Beispiel ist der Rechtsakt zur Arbeitszeitregulierung im Bereich des Luftverkehrs nicht im EUR-Lex 7 rubriziert. 39 Rechtsakte waren in beiden Bereichen gespeichert. Nach Bereinigung der Duplikate ergibt sich die Gesamtzahl von 280 relevanten Rechtsakten. Siehe Plehwe: Transformation Europäischer Governance, 2007, S. 52-54. 
Wird über die Differenzierung zwischen bindendem und nicht bindendem Recht in Grafik 8 hinaus zwischen den verschiedenen Typen des bindenden Rechts unterschieden, dann zeigt sich eine deutliche Vorherrschaft von Richtlinien (Grafik 9).

\section{Grafik 9: Europäische Verkehrssozialrechtsetzung 1960-2004, hard law}

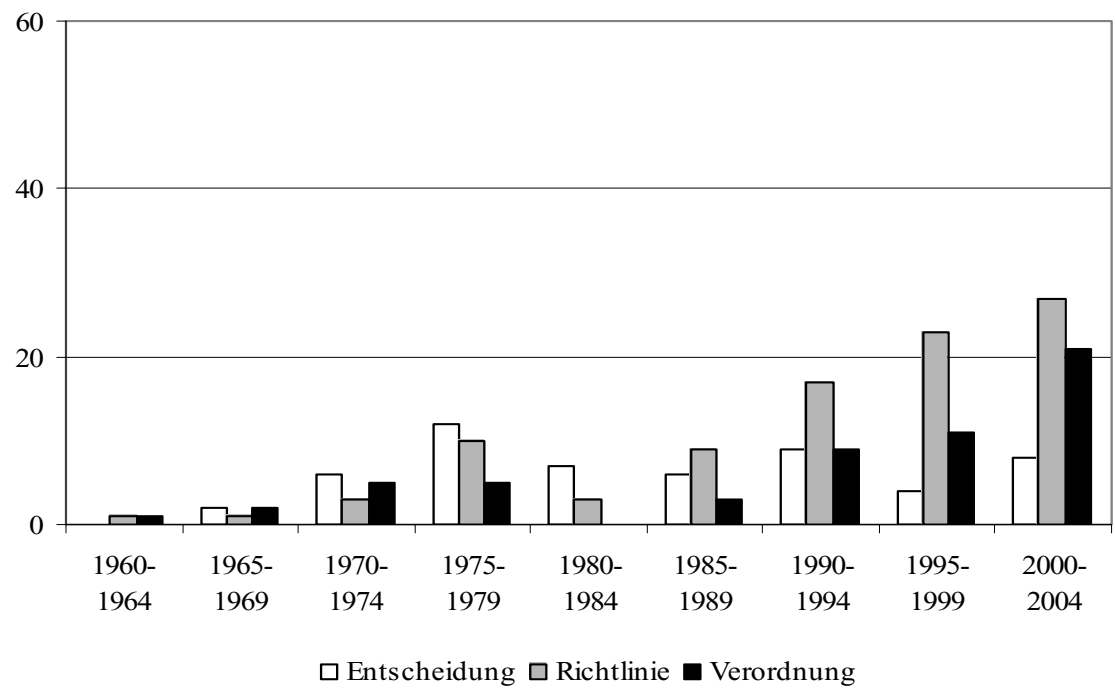

Quelle: EUR-Lex 7 und 5 (Stand Dezember 2005), Fünfjahresintervalle, eigene Berechnung.

Allerdings stieg in der jüngeren Zeit die Zahl der Verordnungen in diesem Bereich erheblich rascher als die Zahl der Richtlinien. Tatsächlich überstieg im jüngsten hier betrachteten Zeitraum (2002-2004) die Zahl der Verordnungen jeweils die Zahl der Richtlinien. ${ }^{27}$ Mithin deutet sich möglicherweise im Bereich der Verkehrssozialpolitik ein Wandel im Europäisierungsmodus an. Die vorwiegende Verwendung von Verordnungen legt die Hypothese nahe, dass der prinzipiell nationalstaatlich vermittelte Europäisierungstyp durch einen stärker supranational ausgerichteten Entwicklungsmodus abgelöst wird. Weil sich im Bereich der Verkehrsumweltpolitik eine ähnliche Entwicklung zeigt, lässt sich darüber hinaus die Hypothese aufstellen, dass es zu einer Homogenisierung der Goverance-Typen und Europäisierungsmodi im Politikfeld Verkehr kommt - vorausgesetzt natürlich, die Entwicklung der vergangenen Jahre setzt(e) sich ohne große Änderungen fort.

Im Bereich der Verkehrsumweltpolitik zeigen die Angaben in Grafik 10 ein seit Anfang und insbesondere seit Mitte der 1990er Jahre starkes Wachstum des bindenden Rechts.

Im Vergleich zum Sozialrecht spielten nicht bindende Regeln in der Umweltpolitik im Politikfeld Verkehr keine große Rolle. Lediglich in der ersten Hälfte der 1980er Jahre spielten nicht bindende Regeln eine dem bindenden Recht vergleichbare und gleichermaßen geringe Rolle. Ebenfalls anders als in der Sozialpolitik wurde im bindenden Recht bis in die

27 Auch wenn Grafik 9 für das letzte Fünfjahresintervall insgesamt eine höhere Zahl von Richtlinien darstellt, überstieg die Zahl der Verordnungen bereits seit 2002 die Zahl der erlassenen Richtlinien (2002: fünf Richtlinien, sechs Verordnungen; 2003: fünf Richtlinien, neun Verordnungen; 2004: drei Richtlinien, vier Verordnungen). 
2000er Jahre nahezu ausschließlich mit Richtlinien gearbeitet (siehe Grafik 11). Aber auch in diesem Bereich stieg die Zahl der Verordnungen in der jüngsten Zeit stark an.

\section{Grafik 10: Europäische Verkehrsumweltrechtsetzung 1960-2004}

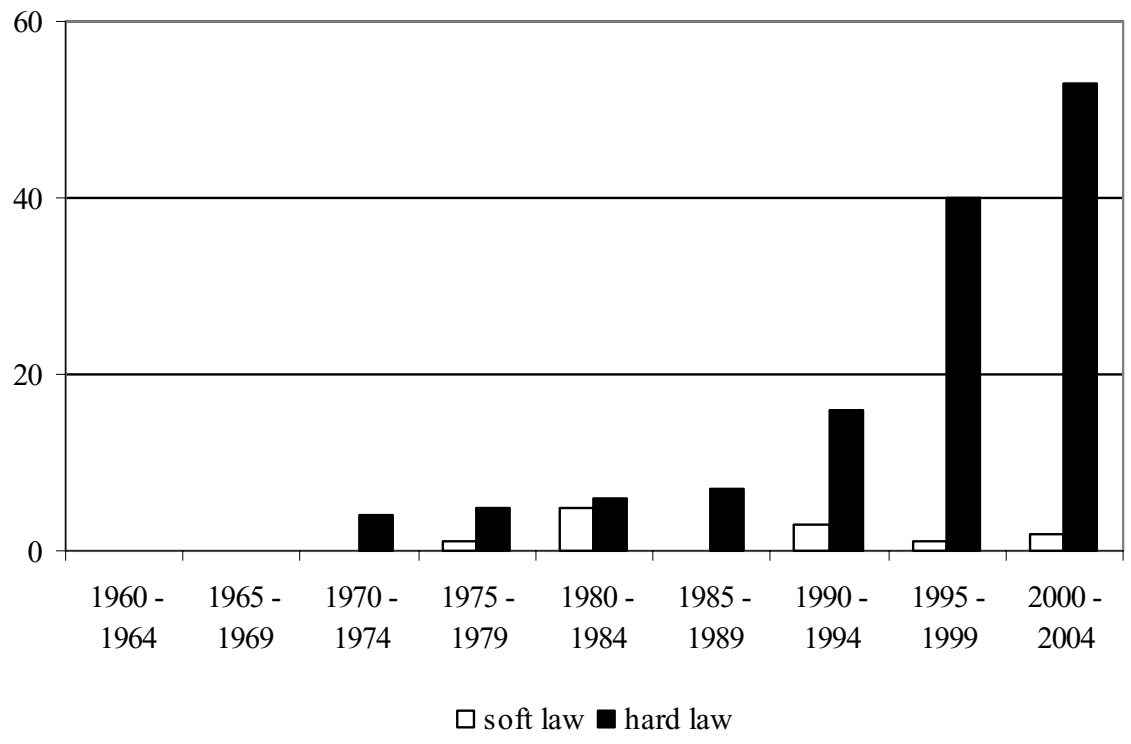

Quelle: EUR-Lex 7 und 15 (Stand Dezember 2005), Fünfjahresintervalle, eigene Berechnung.

Grafik 11: Europäische Verkehrsumweltrechtsetzung 1960-2004, hard law

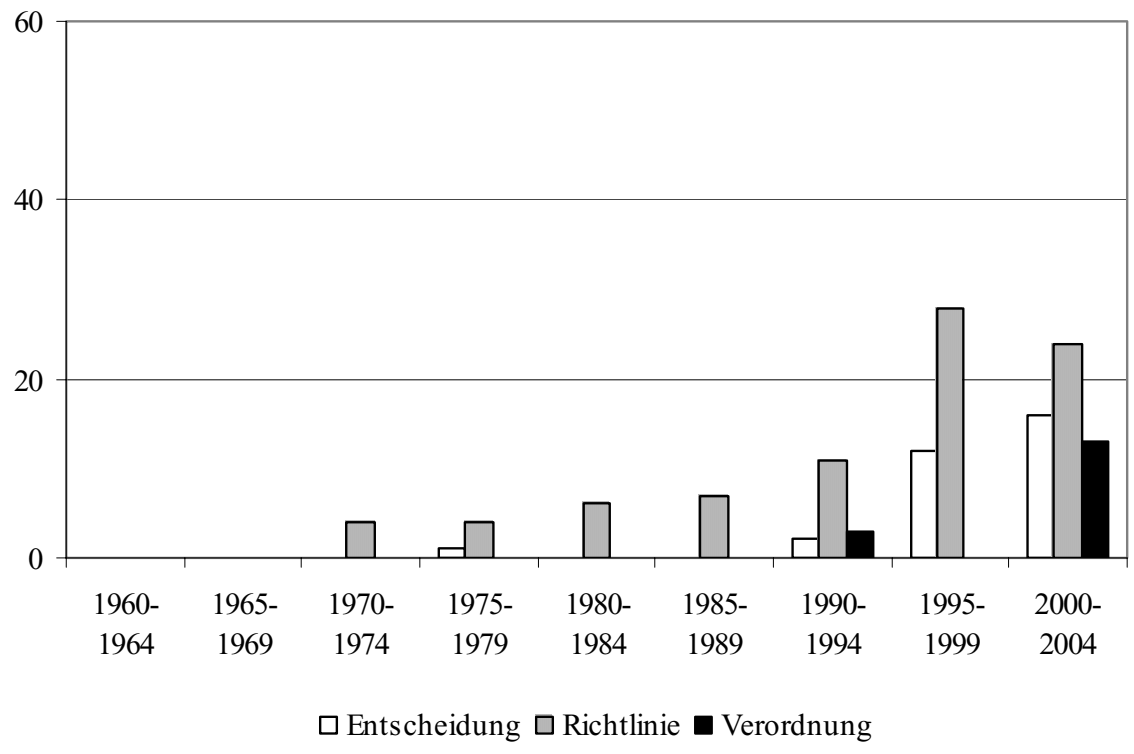

Quelle: EUR-Lex 7 und 15 (Stand Dezember 2005), Fünfjahresintervalle, eigene Berechnung. 
Weil in der jüngsten Zeit (2003 und 2004) die Zahl der Verordnungen gegenüber der Zahl der Richtlinien überwog, ${ }^{28}$ wird auch in der Verkehrsumweltpolitik möglicherweise ein stärker supranational gelenkter Europäisierungsprozess angezeigt.

\section{Quantitative Verfahren: brauchbare Hypothesen zu vorherrschenden Governance- Typen und Europäisierungsmodi?}

Anhand der quantitativen Analyse des europäischen Rechts im Politikfeld Verkehr konnte gezeigt werden, dass dieses Politikfeld im Hinblick auf Verkehrsträger und -branchen sowie verschiedene Regelungsmaterien (Marktordnungs-, Sozial- und Umweltpolitik im Verkehr) sukzessive durch eine mittlerweile alle wichtigen Bereiche der Verkehrspolitik erfassende europäische Rechtsetzung europäisiert wurde. Gleichzeitig erlaubt die Unterscheidung von Governance-Formen anhand verschiedener Arten von Rechtsakten (bindendes und nicht bindendes Recht sowie Verordnungen und Richtlinien) die Verdeutlichung von wichtigen Unterschieden. Deshalb muss auf den heterogenen Charakter der Europäisierung hingewiesen werden. Während sich ,Koordination" und ,Hierarchie“ als GovernanceTypen im Verkehr bis in die 1970er Jahre hinein in etwa die Waage hielten, erlangte seither das bindende Recht gegenüber dem nicht bindenden Recht klar Vorrang. Eine hinreichend differenzierte Analyse bringt dabei zum Vorschein, dass in einzelnen Branchen und Regelungsmaterien in erster Linie supranational verbindliche Verordnungen verabschiedet werden (zum Beispiel Marktordnungsrechtsakte im Straßengüterverkehr und Luftverkehr), während in der Marktordnungspolitik im Eisenbahnverkehr sowie in der Verkehrssozialund der Verkehrsumweltpolitik überwiegend Richtlinien zum Einsatz kommen. Also lässt ein verbindliches europäisches Rahmenrecht unterschiedlich große Spielräume für nationale Anpassungen zu. Weil in jüngster Zeit beispielsweise auch sozial- und umweltpolitische Belange im Verkehr überwiegend mit Verordnungen geregelt werden, kann unterdessen die Hypothese entwickelt werden, dass nach der weitgehenden Durchsetzung einer europäischen Markt- und Wettbewerbsordnung nationalstaatliche Spielräume in weiteren verkehrspolitischen Belangen verringert werden (sollen). Generell erscheint das Instrument der quantitativen Analyse der Rechtsentwicklung als nützlich, um Hypothesen zu vorherrschenden Governance-Typen und Europäisierungsmodi beziehungsweise zu Veränderungen des Europäisierungsprozesses zu entwickeln.

Allerdings ist im Falle einer quantitativen Analyse des europäischen Rechts besondere Vorsicht geboten, weil die zugrunde liegenden Datensätze, die offizielle EUR-Lex-Klassifikation, viele Probleme aufwirft. Die offizielle Klassifikation entspricht nur bedingt einer angemessenen Einteilung von Politikfeldern, zumindest was die Verkehrspolitik angeht. Nur eine systematische Ergänzung der verkehrspolitisch relevanten Rechtsakte des Wettbewerbs-, Umwelt-, Sozial-, Infrastruktur- und Technologierechts verringert die Gefahr einer nicht sachgerechten Deflation des europäischen Verkehrsrechts auf der Basis der offiziellen Klassifikation. Immerhin knapp 200 Rechtsakte konnten unter anderem anhand einschlägiger Eurovoc-Deskriptoren (Schlagwort Transport etc.) in anderen Klassifikationsbereichen (nach Einzelprüfung) als verkehrspolitisch relevant identifiziert werden, also etwa 20 Prozent der offiziellen verkehrspolitischen Rechtsetzung ( $\mathrm{N}=965)$ gemäß EUR-Lex 7 , Ver$\mathrm{kehr}^{6}$. Gleichzeitig muss aus anderen Gründen die Gefahr einer Inflation der Verkehrsrecht-

28 Ähnlich wie bei Grafik 9 verbirgt eine Gesamtbetrachtung der Rechtsetzung in fünf Jahren, dass die Zahl der Verordnungen gegenüber der Zahl der Richtlinien in den beiden letzten Jahren des Zeitraums überwog. Die Einzelzählung ergibt für das Jahr 2003 drei Richtlinien und fünf Verordnungen und für das Jahr 2004 drei Richtlinien und vier Verordnungen. 
setzung erkannt und gebannt werden, weil Unterschiede zwischen den verschiedenen Typen des bindenden und des nicht bindenden Rechts eine einheitliche Zählung der Rechtsakte verbieten. Im Bereich des besonders relevanten bindenden Rechts können beispielsweise Entscheidungen, Richtlinien und Verordnungen nur bedingt miteinander verglichen werden, weil Entscheidungen meist nur für bestimmte Mitgliedstaaten Geltung erlangen.

Über die Notwendigkeit der systematischen Differenzierung und Untersuchung der Teilpolitikfelder und der Arten von Rechtsakten hinaus ist bei quantitativen Verfahren zu berücksichtigen, dass eine große Zahl von Rechtsakten keineswegs notwendigerweise auf eine besonders starke oder intensive Europäisierung verweisen muss. Anhand der europäischen Arbeitszeitrechtsetzung im Verkehr wurde darauf hingewiesen, dass genau das Gegenteil der Fall sein kann: Ein einziger Rechtsakt regelt europäische Mindeststandards für alle Arbeitnehmer/-innen mit Ausnahme des Verkehrs, wohingegen die diesbezügliche Lücke im Verkehrssektor mit zum Teil die Standards verwässernden Regeln jeweils für die einzelnen Branchen geschlossen werden mussten. Schließlich muss nachdrücklich betont werden, dass quantitative Verfahren nicht an die Stelle von qualitativen Überlegungen treten sollten. Die gemeinsame Verkehrspolitik eignet sich hervorragend zur Erläuterung des sogenannten ,Degreeism'-Problems: Während die europäische Rechtsetzung bis Mitte der 1980er Jahre vorwiegend darauf abzielte, sozial- und technologiepolitische Vorraussetzungen für eine gemeinsame Verkehrspolitik zu schaffen (Harmonisierung), wurde die europäische Verkehrsrechtsetzung im Bereich der Marktordnung seit Mitte der 1980er Jahre durch ein Urteil des Europäischen Gerichtshofes und das folgende Binnenmarktprogramm dynamisiert (Liberalisierung). $\mathrm{Zu}$ beachten und gegebenenfalls zu messen sind also nicht nur Governance-Formen und Europäisierungsgrade, sondern möglicherweise auch sehr unterschiedliche Inhalte der Europäisierung (regulierter Markt oder Wettbewerbsmarkt etc.).

Trotz vieler und manchmal schwerwiegender Vorbehalte sowohl im Hinblick auf quantitative als auch qualitative Fragen erscheint der Einsatz quantitativer Verfahren in der (vergleichenden) Europaforschung aber in anderer Hinsicht durchaus als nützlich und vielversprechend, weil eine auf Institutionen und geltende (Entscheidungs-)Verfahren verengte Analyse die Gefahr birgt, eine ganze Reihe von Europäisierungsdynamiken zu verkennen. So entwickelte sich die Europäisierung in einigen Bereichen der Verkehrspolitik bereits vor der Einführung von Mehrheitsentscheidungen oder sogar unabhängig davon sehr dynamisch, weil andere Faktoren (Entscheidungen des Europäischen Gerichtshofes, spezifische Kompetenzen der Kommission etc.) dafür sorgten, dass retardierende Veto-Positionen aufgegeben werden mussten. Ob sich anhand der Vorherrschaft von Richtlinien oder Verordnungen Hypothesen zur Intensität und Reichweite der Europäisierung bewähren (analog zur noch stark nationalstaatlich geprägten Marktordnungspolitik im Bereich der Eisenbahnen im Vergleich zum weitgehend supranational geregelten Straßengüterverkehr), muss und kann anhand von weiteren Politikfeldern geprüft werden. Allerdings ist ein hinreichendes Verständnis des Politikfeldes und der (Europa-)Rechtsmaterie unabdingbar, weil die erforderliche Grundlage relevanter ,Rechtsdaten " nicht im blinden Vertrauen auf die offizielle Klassifikation des EUR-Lex geschaffen werden kann. 